\title{
HUELLA DE CARBONO EN CINCO ESTABLECIMIENTOS DE SALUD DEL TERCER NIVEL DE ATENCIÓN DE PERÚ, 2013
}

\author{
Celso Bambarén-Alatrista ${ }^{1,2, a}$, María del Socorro Alatrista-Gutiérrez $z^{3,4, b}$
}

\begin{abstract}
RESUMEN
Se realizó un estudio para calcular la huella de carbono generada por los establecimientos de salud del tercer nivel de atención ubicados en la ciudad de Lima, Perú, en el año 2013. Se obtuvieron los reportes del consumo de recursos energéticos y agua, así como la generación de residuos de cinco establecimientos, los cuales contribuyeron al cambio climático con la emisión de 14462 teq $\mathrm{CO}_{2}$. El 46\% de estas emisiones están asociadas al consumo de combustible para el funcionamiento de la casa de fuerza, generadores eléctricos y vehículos de transporte. Un $44 \%$ se relacionan con el consumo de energía eléctrica, y el restante $10 \%$ con la utilización de agua y generación de residuos sólidos hospitalarios. $\mathrm{CO}_{2}, \mathrm{~N}_{2} \mathrm{O}$ y $\mathrm{CH}_{4}$ son los gases de efecto invernadero incluidos en la estimación de la huella de carbono. Los hospitales tienen un impacto ambiental negativo, principalmente debido al consumo de combustibles fósiles.
\end{abstract}

Palabras clave: Hospitales, Huella de carbono, Cambio climático (fuente: DeCS BIREME).

\section{CARBON FOOTPRINT IN FIVE THIRD-LEVEL HEALTH CARE CENTERS IN PERU, 2013}

\begin{abstract}
This study was performed to calculate the carbon footprint generated by third-level health care centers located in Lima, Peru, in 2013. Reports were obtained on the consumption of energy resources and water as well as on waste generation from the five centers, which contributed to climate change with an emission of 14,462 teq of $\mathrm{CO}_{2}$. A total of $46 \%$ of these emissions were associated with fuel consumption by the powerhouse, power generators, and transport vehicles; $44 \%$ was related to energy consumption; and the remaining $10 \%$ was related to the use of water and generation of solid hospital waste. $\mathrm{CO}_{2}, \mathrm{~N}_{2} \mathrm{O}$, and $\mathrm{CH}_{4}$ are the greenhouse gases included in the estimated carbon footprint. Our results show that hospitals have a negative environmental impact, mainly due to fossil fuel consumption.
\end{abstract}

Key words: Hospitals, Carbon footprint, Climate change (source: MeSH NLM).

\section{INTRODUCCIÓN}

Ante el cambio climático, el sector Salud enfrenta dos retos, el diseño de intervenciones para atenuar las consecuencias de este cambio en la salud y prevenir la incidencia de enfermedades, así como la implementación de las medidas para mitigar el efecto negativo de los establecimientos de salud en el medioambiente.

El sector Salud genera emisiones, principalmente por el uso energético de los edificios, por la generación y transporte de materiales, transporte de pacientes, uso de equipos, y la generación y transporte de residuos. También, se pueden deber por el mal funcionamiento de equipos (emisiones fugitivas por pérdidas de gases refrigerantes en equipos de aire acondicionado), a los procesos químicos (para depuración del agua) y las emisiones derivadas del uso de gases medicinales y anestésicos, debido a que estos no son metabolizados por el organismo y son exhalados en pocos minutos.

El Servicio Nacional de Salud (NHS) del Reino Unido genera emisiones que ascienden a más de 18 millones de toneladas de $\mathrm{CO}_{2} / \mathrm{año}$, que supone un $25 \%$ de las emisiones totales del sector público en este país ${ }^{(1)}$. Además, el $5 \%$ del total de emisiones asociadas al transporte corresponden al NHS debido a los viajes de personal, pacientes y visitantes, de los cuales $83 \%$ son producidas por coche o furgoneta ${ }^{(2)}$. El consumo energético de los centros hospitalarios de la Comunidad de Madrid se centra $45 \%$ en climatización, $35 \%$ en iluminación y $20 \%$ en producción de agua caliente sanitaria ${ }^{(3)}$. Un estudio de 15 servicios de emergencia

\footnotetext{
Universidad Peruana Cayetano Heredia. Lima, Perú

Universidad de San Martín de Porres. Lima, Perú

Universidad Nacional Mayor de San Marcos. Lima, Perú

Universidad Ricardo Palma. Lima, Perú

Médico cirujano; b. doctor en medicina

Recibido: 10/01/2016 Aprobado: 09/03/2016
}

Citar como: Bambarén-Alatrista C, Alatrista-Gutiérrez MdS. Huella de carbono en cinco establecimientos de salud del tercer nivel de atención de Perú, 2013. Rev Peru Med Exp Salud Publica. 2016;33(2):274-7. doi:10.17843/rpmesp.2016.332.2141 
en los Estados Unidos de Norteamérica encontró que el $75 \%$ de las emisiones provenían del diésel o gasolina utilizadas en los vehículos empleados para la atención de emergencias ${ }^{(4)}$.

Para diseñar las medidas de mitigación, se requiere estimar su huella de carbono, la cual describe la cantidad de emisiones de gases de efecto invernadero (GEI) que son liberadas a la atmósfera por una organización, producto, servicio u obra ${ }^{(5)}$. La finalidad del estudio fue la estimación de la huella de carbono generada por el funcionamiento de establecimientos de salud del mayor nivel de complejidad, categorizados en el tercer nivel de atención ${ }^{(6)}$.

\section{MATERIALES Y MÉTODOS}

El estudio fue descriptivo y transversal destinado a la estimación de la huella de carbono para el año 2013, de los establecimientos del Ministerio de Salud categorizados como III ubicados en Lima Metropolitana.

En primer lugar, se revisó el reporte anual de ecoeficiencia de las entidades públicas ${ }^{(7)}$, el cual contiene información sobre el consumo anual de agua, energía eléctrica y combustible. En segundo lugar, se buscaron los reportes de ecoeficiencia ${ }^{(8)}$ en las páginas web de los establecimientos de salud. La cantidad de residuos sólidos generados se obtuvo de los planes de manejo de residuos sólidos hospitalarios.

El estudio incluyo los tres alcances para la medición de la huella de carbono ${ }^{(9)}$. El primero, sobre las emisiones directas de $\mathrm{GEI}$, que están asociadas a fuentes que están bajo el control de los establecimientos de tipo fijas (calderas, grupos electrógenos) o móviles ${ }^{(10)}$ como vehículos y ambulancias. Se incluyó información sobre el consumo anual de los combustibles fósiles utilizados en los calderos y en los vehículos como diésel, GLP y la gasolina de 84,90 y 97 octanos. No se consideró las emisiones fugitivas y asociadas a gases medicinales y anestésicos.
El segundo alcance correspondió a las emisiones indirectas que no están bajo el control del establecimiento, y están asociadas a la adquisición y consumo de la energía eléctrica por la organización ${ }^{(10)}$. El tercer alcance incluyó otras emisiones indirectas producidas por actividades o procesos que forman parte de la cadena de valor del establecimiento, pero que están fuera de los límites organizacionales, en este alcance se seleccionó la generación de residuos sólidos y la utilización del agua potable ${ }^{(10)}$.

Con base en los valores de los consumos y generación de residuos obtenidos de cada establecimiento, se realizó una medición intermitente para el año 2013, obteniéndose la huella de carbono total expresada en toneladas equivalentes de $\mathrm{CO}_{2}$ (teq $\mathrm{CO}_{2}$ ), que es la sumatoria de la multiplicación de los datos de las actividades de GEI por los factores de emisión de GEI. Estos factores fueron obtenidos del Ministerio de Energía y Minas para la energía eléctrica, diésel y los diferentes octanajes de gasolinas ${ }^{(11)}$, y de la guía de la oficina Catalana de cambio climático para el GLP, consumo de agua y la generación de residuos sólidos sin segregar ${ }^{(12)}$.

\section{RESULTADOS}

Se encontró información en el reporte anual de ecoeficiencia de las entidades públicas ${ }^{(7)}$ sobre el Instituto Nacional de Enfermedades Neoplásicas (INEN), Hospital de Emergencias Pediátricas (HEP) y el Hospital Nacional Docente Madre-Niño San Bartolomé (HNSB), y en sus respectivas páginas web acerca del INEN, Instituto Nacional de Salud del Niño - sede Jesús María (INSN) y el Hospital Nacional Hipólito Unanue (HNHU).

En la Tabla 1, se resume la información obtenida sobre cada uno de los cinco establecimientos de salud. Se observa que el mayor consumo de energía eléctrica corresponde al INEN y en segundo lugar al INSN. Cuando se compara según $\mathrm{kWh} /$ cama/día, el rango de consumo va de 7 a 30, donde el mayor valor corresponde al HEP

Tabla 1. Consumo de recursos energéticos, agua y generación de residuos sólidos en cinco establecimientos de salud del tercer nivel de atención en Lima, Perú 2013

\begin{tabular}{|c|c|c|c|c|c|c|c|c|}
\hline $\begin{array}{l}\text { Establecimiento } \\
\text { de salud }\end{array}$ & $\begin{array}{c}\text { Gasolina } \\
84 \\
\text { octanos } \\
\text { (I) }\end{array}$ & $\begin{array}{c}\text { Gasolina } \\
90 \\
\text { octanos } \\
\text { (I) }\end{array}$ & $\begin{array}{c}\text { Gasolina } \\
97 \\
\text { octanos } \\
\text { (I) }\end{array}$ & $\begin{array}{c}\text { GLP } \\
\text { (I) }\end{array}$ & $\begin{array}{c}\text { Diésel } \\
2 \text { (I) }\end{array}$ & $\begin{array}{c}\text { Consumo } \\
\text { de energía } \\
\text { eléctrica } \\
\text { (kWh) }\end{array}$ & $\begin{array}{c}\text { Consumo } \\
\text { de agua } \\
\left(\mathrm{m}^{3}\right)\end{array}$ & $\begin{array}{l}\text { Generación } \\
\text { de residuos } \\
\text { sólidos } \\
\text { (t) }\end{array}$ \\
\hline INEN & 0 & 4568 & 0 & 17596 & 6347 & 3132048 & 119561 & 419 \\
\hline INSN & 814 & 5912 & 5640 & 0 & 350504 & 3106332 & 182318 & 820 \\
\hline HEP & 0 & 0 & 0 & 0 & 3485 & 633248 & 15070 & 82 \\
\hline HNSB & 0 & 0 & 0 & 0 & 0 & 1671149 & 78756 & 120 \\
\hline $\mathrm{HNHU}$ & 0 & 95 & 0 & 0 & 309916 & 1683368 & 162173 & 621 \\
\hline Total & 814 & 10575 & 5640 & 17596 & 670252 & 10226145 & 557878 & 2062 \\
\hline
\end{tabular}

INEN: Instituto Nacional de Enfermedades Neoplásicas; INSN Instituto Nacional de Salud del Niño - sede Jesús María; HEP: Hospital de Emergencias Pediátricas y HNSB; Hospital Nacional Docente Madre-Niño San Bartolomé; HNHU: Hospital Nacional Hipólito Unanue. 
seguido del INEN. El mayor consumo total de agua correspondió al INSN seguido del HNHU. En unidades litro/cama/día, los valores van de 677 a 977, el INSN tiene el mayor gasto seguido del HNSB. EI INSN produce la mayor cantidad estimada de residuos sólidos con 820 toneladas por año seguido de las $621 \mathrm{t}$ del HNHU.

Los valores de los recursos consumidos y los residuos generados se multiplicaron por los factores de emisión, tomando en cuenta las unidades en estaban expresados, lo que da como resultado la emisión de toneladas equivalentes de $\mathrm{CO}_{2}$ en un año. En la Tabla 2 se muestra que el total de emisiones es de 14462 toneladas para los CINCO establecimientos de salud. El mayor impacto sobre el medioambiente corresponde al INSN debido a que emite 5992 teq $\mathrm{CO}_{2}$ seguido del HNHU con 4494 y el INEN en tercer lugar. El consumo de combustibles fósiles que forman parte del alcance 1, contribuye en mayor medida a las emisiones totales.

En la Figura 1 se presenta la distribución, según alcances, de las emisiones emitidas, donde el $46 \%$ de estas emisiones están asociadas al consumo de gasolina de diversos octanajes, diésel y GLP, que se utilizan en la casa de fuerza, generadores eléctricos y vehículos de transporte. El consumo de energía eléctrica le corresponde al $44 \%$, y el restante $10 \%$ a la generación de residuos sólidos hospitalarios y la utilización de agua potable.

\section{DISCUSIÓN}

Los cinco establecimientos de salud generan más de 14 mil teqCO $\mathrm{CO}_{2}$ con un valor promedio anual de 2400 , lo cual supera el promedio de algunos hospitales en otros países que producen 1500 toneladas ${ }^{(14)}$. Los resultados muestran que la cantidad de teq $\mathrm{CO}_{2}$ no guardan una relación directa con el número de camas o la complejidad de los servicios que prestan. EI INSN y el HNHU son aquellos que producen una mayor huella de carbono, mientras que el INEN que cuentan con equipos tecnológicos de mayor complejidad se ubica en el tercer lugar.
Similar situación se presenta con el consumo de energía eléctrica, donde el HEP está en el primer lugar de gasto, superando a establecimientos de mayor complejidad tecnológica y con mayor número de camas como el INEN e INSN. El gran consumo de estos recursos puede estar asociado a fugas de agua, mal funcionamiento de equipos e instalaciones, requerimientos mayores para la operación de equipos, y el uso intensivo de medios de climatización e iluminación por no contar con una buena ventilación e iluminación natural.

El consumo energético se reparte entre energía eléctrica (emisiones directas) y térmica (emisiones indirectas) que se utiliza para climatización, calentamiento del agua, iluminación y la operación de equipos tecnológicos (15). Se encontró que las emisiones directas son las que más contribuyen a la huella de carbono, resultado que difiere de una investigación donde el mayor valor correspondió a las emisiones indirectas con más del $55 \%$ del total ${ }^{(13)}$. Esta situación podría estar explicada porque cuatro establecimientos hacen un uso intensivo del diésel y gasolina, productos que tienen un alto factor de emisión, excepto el INEN que consume principalmente GLP, que tiene un factor menor en al menos cuatro veces que los otros combustibles.

Las emisiones del alcance 2 son mayores en el INEN y en el INSN, lo cual estaría vinculado a que estos establecimientos tienen equipos de gran complejidad tecnológica que requieren un mayor suministro de energía. Los resultados del alcance 3 pueden disminuir si se realiza la segregación de los residuos sólidos en vidrio, papel, envases y materia orgánica, debido a que los factores de emisión específicos de cada uno de ellos son menores que cuando se utilizan los residuos sin segregar.

Para elaborar el estudio se obtuvieron los datos de los reportes de ecoeficiencia ${ }^{(8)}$, aunque solo se obtuvo información del $26 \%$ del total de establecimientos del tercer nivel de atención pertenecientes al Ministerio de Salud en la ciudad de Lima. Además, no se incluyeron las emisiones por gases medicinales y anestésicos; estos

Tabla 2. Emisiones generadas por cinco establecimientos de salud del tercer nivel de atención expresadas en teq $\mathrm{CO}_{2}$ según fuentes. Lima, Perú 2013

\begin{tabular}{lccrrr}
\hline $\begin{array}{l}\text { Establecimientos } \\
\text { de salud }\end{array}$ & $\begin{array}{c}\text { Combustibles } \\
\text { fósiles }\end{array}$ & Energía eléctrica & Agua & Residuos sólidos & $\begin{array}{c}\text { Total } \\
\text { teq CO }\end{array}$ \\
\hline INEN & 125,64 & 1926,21 & 47,23 & 261,54 & 2360,62 \\
INSN & 3497,58 & 1910,39 & 72,02 & 512,46 & 5992,45 \\
HEP & 33,80 & 389,45 & 5,95 & 51,41 & 480,61 \\
HNSB & 0 & 1027,76 & 31,11 & 74,97 & 1133,84 \\
HNHU & 3006,93 & 1035,27 & 64,06 & 388,08 & 4494,34 \\
Total & 6663,95 & 6289,08 & 220,37 & 1288,46 & 14461,86 \\
\hline
\end{tabular}

INEN: Instituto Nacional de Enfermedades Neoplásicas; INSN Instituto Nacional de Salud del Niño - sede Jesús María; HEP: Hospital de Emergencias Pediátricas y HNSB; Hospital Nacional Docente Madre-Niño San Bartolomé; HNHU: Hospital Nacional Hipólito Unanue. Teq; Toneladas equivalentes de CO 2 


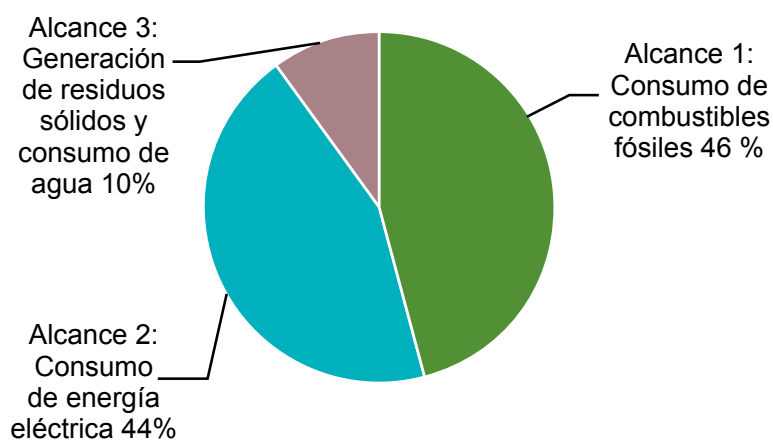

Figura 1. Distribución en porcentajes, de las emisiones según alcances de la medición de la huella de carbono en cinco hospitales del tercer nivel de atención. Lima, Perú. 2013.

últimos tienen un gran impacto, debido a su alto factor de emisión. A ello se suma la falta de información de las emisiones indirectas producidas por el desplazamiento de los visitantes, pacientes y personal, y el dióxido de carbono emitido por la adquisición de bienes y servicios.
Estos valores son difíciles de calcular, pero pueden ser unas a tres veces mayores que las emisiones directas ${ }^{(14)}$.

Los resultados del estudio muestran la contribución de los establecimientos de salud al cambio climático debido a que en estas instalaciones están presentes cuatro de los GEI, y se realizan procesos que utilizan recursos y genera residuos, algunos de los cuales con altos factores de emisión. La estimación de la huella de carbono es un primer paso para sensibilizar a las autoridades sobre la necesidad de implementar medidas de mitigación para reducir el impacto negativo de este tipo de establecimientos en el medioambiente.

Contribuciones de autoria: CB y MdSA han participado en la concepción del artículo, la recolección y análisis de datos, su redacción y aprobación de la versión final.

Fuentes de financiamiento: autofinanciado.

Conflictos de interés: los autores declaran no tener conflictos de interés.

\section{REFERENCIAS BIBLIOGRÁFICAS}

1. National Health Service (UK). Saving carbon, improving Heath. NHS carbon reduction strategy. Cambridge: NHS; 2009.

2. Mayor S. NHS should take steps to cut its carbon footprint, BMA says. BMJ. 2008;336(7647):740. doi: 10.1136/ bmj.39538.375706.DB

3. Comunidad de Madrid. Consejería de Economía y Hacienda. Guía de ahorro y eficiencia energética. Madrid: La Comunidad de Madrid; 2010.

4. Blanchard IE, Brown L, North American EMS Emissions Study Group. Carbon Footprinting of North American Emergency Medical Services Systems. Prehosp Emerg Care. 2011;15(1):23-9. doi: 10.3109/10903127.2010.519818.

5. Alcaldía Mayor de Bogotá. Secretaria distrital de ambiente. Subdirección de políticas y planes ambientales. Guía para la elaboración del informe de huella de carbono corporativa en entidades públicas del distrito capital. Bogotá: Alcaldía Mayor de Bogotá; 2013.

6. Ministerio de Salud del Perú. Categorías de establecimientos del sector salud. NTS 021-MINSA/DGSP-v.03. Lima: MINSA; 2011
7. Ministerio del Ambiente del Perú. Instituciones públicas ecoeficientes. Informe Anual 2013. Lima: MINAM; 2013.

8. Ministerio del Ambiente del Perú. Medidas de ecoeficiencia para el sector público. Decreto Supremo 009-2009-MINAM. Lima:MINAM; 2013.

9. Ministerio de Agricultura, Alimentación y Medio Ambiente de España. Oficina Española de Cambio Climático. Guía para el cálculo de la huella de carbono y para la elaboración de un plan de mejora de una organización. Madrid: Ministerio de Agricultura, Alimentación y Medio Ambiente; 2015.

10. Servicio de Protección Ambiental (SEPA). Vicerrectorado de Coordinación Institucional e Infraestructuras. Universidad de Córdoba. La huella de Carbono de la Universidad de Córdoba 2013. Córdova: SEPA-UCO; 2014.

11. Ministerio de Energía y Minas del Perú. Dirección general de eficiencia energética. Uso eficiente de la energía. Guía metodológica para docentes de secundaria. Ficha informativa 2. La huella de carbón y la eficiencia energética. Lima: MINEM; 2013.
12. Oficina Catalana del Canvi Climàtic. Guia pràctica per al càlculd'emissions de gasos ambefected' hivernacle. Cataluña: Oficina Catalana del Canvi Climàtic; 2015.

13. Arrechea-Enériz R, Aldea-Jimeno A, González Gutiérrez R, Jimeno Llerena R, Peñalva Segura PJ. Inventario de gases efecto invernadero en un hospital. Ingeniera Hoy. 2012;12:4-12.

14. SANITAS. Informe de gases de efecto invernadero en hospitales de participación pública privada. Madrid: SANITAS; 2014.

15. Health without harm. Addressing climate change in the healthcare setting: opportunities for action. Reston: Health without farm; 2009.

Correspondencia: Celso Bambaren Alatrista Dirección: Loma Verde 130, Lima 33, Perú. Teléfono: (+511) 999040762

Correo electrónico: celso.bambaren.a@upch.pe 